\title{
Modeling of Magneto-Mechanical Response of Magnetorhological Elastomers having Different Arrangement of Magnetic Particles
}

\author{
Mostafa Asadi Khanouki and Ramin Sedaghati \\ Department of Mechanical, Industrial and Aerospace Engineering \\ Concordia University \\ Montreal, QC, Canada
}

\begin{abstract}
A model describing the magneto-mechanical properties of magnetorheological elastomers (MREs) under an external magnetic field and a mechanical shear deformation is presented. The main purpose of the present study is to demonstrate the effect of particle distribution and applied magnetic field on the MRE mechanical properties. Four types of rectangular lattice models are considered as the representation of spatial distribution of magnetic particles in the matrix. Using the energy method, shear modulus is obtained and numerically calculated as function of the strength of the external magnetic field and the shear strain. The results show a high sensitivity of shear modulus on the spatial distribution of particles. Depending on the lattice type, shear modulus exhibits an increasing or decreasing behavior with the increase of magnetic field intensity.
\end{abstract}

Keywords- MR elastomers; microstructure modeling; magnetomechanical properties; particle distribution

\section{INTRODUCTION}

Among different types of smart materials, those that are responsive to an external magnetic field are called magnetically activated smart materials or simply magneto-active materials. Basically, magneto-active materials are composed of two main components: magnetizable particles and nonmagnetic matrix. The host nonmagnetic matrix can be a solid or a liquid, which are then impregnated with micron-sized ferromagnetic particles. Once a magnetic field is applied, the rheology of the material will change as a result of magnetic interaction between particles. Therefore, a common name for the magneto-active materials is magnetorheological materials or in abbreviation, MR materials. Among the MR materials, those with a solid elastomeric matrix are called MR elastomer or simply MRE.

MREs can provide field-dependent elastic modulus making them ideal for many applications such as adaptive spring elements in seismic isolators, vibration absorbers, automotive suspension and artificial human body limbs to name a few [1]. Development of physic-based models capable of predicting the mechanical properties of MRES is of paramount importance for design of MRE-based adaptive devices and also development of control strategies. Many theoretical models have been presented to predict magneto-mechanical properties of MREs [2-5]. One of the promising modeling approaches is based on the magnetophysical properties of components of the MREs. The method considers microstructure of the MRE and examines magnetic interaction between magnetic particles and their corresponding effect on elastomeric matrix that causes change of properties under an applied magnetic field [6-9]. Jolly et al. [6] presented a quasi-static dipole model to predict magneto-viscoelastic behavior of MREs with anisotropic (chain-like) distribution of particles. They only considered the interaction of two adjacent dipoles in a chain of particles in their model.

Based on the previous progresses in modeling mechanical behavior of MR fluids [10, 11], Davis [7] has also worked on a dipole model to predict the maximum possible increase of shear modulus (once saturation of MRE is occurred) as a function of saturation magnetization and volume fraction of filler particles. Later, some authors tried to present models to encircle the interactions of all particles in a chain [9] or in the whole structure [12]. As a recent progress in this field, Ivaneyko et al. [8] developed a model that can predict magneto-mechanical behavior of MREs under an applied magnetic field by considering interactions between all particles in the network. They discussed the case of a MRE with isotropic spatial distribution of magnetic particles and finally presented the MRE's modulus of elasticity as a function of magnetic field for tensile deformations.

In the present work, the model of Ivaneyko et al. [8] is extended for the case of static shear deformations in the framework of a nonlinear elasticity approach. Magnetic particles are supposed to be located on the sites of four lattice types namely simple cubic, body centered cubic, face centered cubic and edge centered cubic lattices. The two former lattices were also used in [8] but the two latter ones are utilized here for the first time. In the following, first, the mathematical formulation of the model is introduced. This is followed by the derivation of total potential energy of the deformed MRE in a simple shear test under an applied magnetic field. The field dependent shear modulus is then extracted from the derived energy function. The shear modulus depends on the strength of the magnetic field, shear strain, mechanical and magnetic properties of the matrix and particles and finally on the particles' lattice type. Finally, 
numerical results for the shear modulus are provided and discussed.

\section{MATHEMATICAL FORMULATIONS}

In order to model the mechanical response of a MRE that has a particular spatial distribution of the magnetic particles, the total energy of a MRE sample is obtained in this section. Total energy of the elastomer is a function of the external applied shear deformation, applied magnetic field intensity, lattice model of the magnetic particles and mechanical or magnetic properties of the material. In this study, a MRE sample under a simple shear loading in one direction (x-axis) which is normal to the direction of an applied magnetic field (y-axis) is considered. Fig. 1 illustrates this situation schematically that the simple shear happens in the $\mathrm{x}-\mathrm{y}$ plane and the magnetic field $(H)$ is in the y direction. Magnetic particles inside the sample have magnetic dipoles in the direction of the external field.

\section{A. Total Potential Energy}

Total potential energy of a deformed MRE in the presence of a magnetic field is composed of two parts. One is the elastic energy of the deformed MRE due to the entropic elasticity of polymer chains $\left(e_{e l}\right)$ and the other is due to the potential energy of the magnetic particles placed in an external magnetic field $\left(e_{m}\right)$. Thus, the total potential energy of the deformed $\operatorname{MRE}\left(e_{T}\right)$ under external magnetic field can be written as:

$$
e_{T}=e_{e l}+e_{m}
$$

Here first, the elastic part of the energy $\left(e_{e l}\right)$ is calculated for the case of a simple shear test on a continuous medium. Ogden strain potential is extensively used for such applications which is a nonlinear deformation model and valid for the large strain situations [13]. Here, a simplified form of the Ogden model with known coefficients is utilized. Based on this simplified model, the elastic energy of a MRE sample under nonlinear deformations can be presented with the Neo-Hooke law of the following general form [8]:

$$
e_{e l}=\frac{G_{0}}{2}\left(\lambda_{x}^{2}+\lambda_{y}^{2}+\lambda_{z}^{2}-3\right)
$$

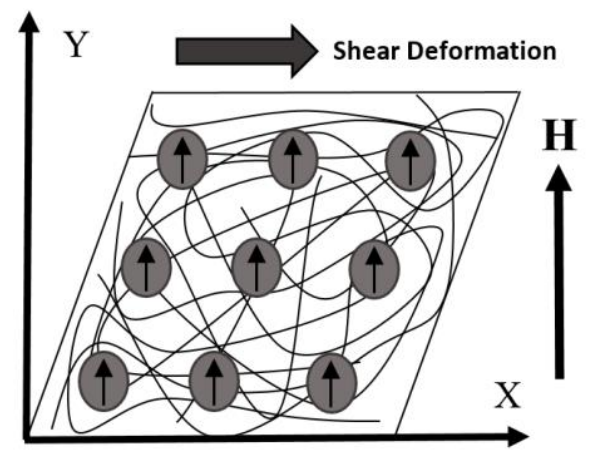

Figure 1. Schematic representation of a MRE sample under simple shear deformation normal to the applied magnetic field direction where $\lambda_{x}, \lambda_{y}, \lambda_{z}$ are the principal extension ratios, i.e. the ratio of the current length to original length, in the principal directions. $G_{0}$ is the shear modulus of the MRE at zero field which is usually obtained experimentally. This material parameter introduces the effect of different complex phenomena into the elastic energy of the sample such as matrix reinforcement by the hard particles, adhesion of the matrix onto the surface of magnetic particles and so forth.

The magneto-mechanical response of the deformed MRE under the applied magnetic field is characterized based on the value of the shear strain $\gamma$ in the $\mathrm{x}-\mathrm{y}$ plane. For the case of large shear strains, the relation between the extension ratio in $x$ direction and the shear strain is [14]:

$$
\gamma=\lambda_{x}-\frac{1}{\lambda_{x}}
$$

Assuming the material to be incompressible $\left(\lambda_{x} \lambda_{y} \lambda_{z}=1\right)$, and considering a plane strain state $\left(\lambda_{y}=1\right)$ the extension ratios are characterized based on the shear strain as:

$$
\begin{gathered}
\lambda_{x}=\frac{\gamma+\sqrt{4+\gamma^{2}}}{2}, \lambda_{y}=1, \\
\lambda_{z}=\frac{1}{\lambda_{x}}=\frac{2}{\gamma+\sqrt{4+\gamma^{2}}}
\end{gathered}
$$

Using relations ( 4 ) into ( 2 ), the elastic energy of the elastomer as a function of $\gamma$ can be expressed as:

$$
e_{e l}=\frac{G_{0}}{2}\left(\left(\frac{\gamma+\sqrt{4+\gamma^{2}}}{2}\right)^{2}+\left(\frac{2}{\gamma+\sqrt{4+\gamma^{2}}}\right)^{2}-2\right)
$$

The second part of the energy in (2) is due to the fieldinduced interaction of magnetic particles inside the elastomer. Applying a magnetic field on the MRE activates the elementary atomic magnetic dipoles in the magnetic particles. The induced dipole moment of each particle has an interaction with the other dipoles in the particle network and is known as the dipoledipole interaction. The magnetic energy of the material which is the dipole-dipole interaction energy per unit volume can be expressed as $[8,15]$ :

$$
\begin{aligned}
e_{m}=-\frac{1}{V} \frac{\mu_{r} \mu_{0}}{4 \pi} \sum_{i j} & {\left[\frac{3\left(\vec{m}_{i} \cdot \vec{R}_{i j}\right)\left(\vec{m}_{j} \cdot \vec{R}_{i j}\right)}{\left|\vec{R}_{i j}\right|^{5}}\right.} \\
& \left.-\frac{\left(\vec{m}_{i} \cdot \vec{m}_{j}\right)}{\left|\vec{R}_{i j}\right|^{3}}\right]
\end{aligned}
$$

where $V$ is the volume of the sample, $\mu_{0}$ is vacuum permeability, and $\mu_{r}$ is the relative permeability of the medium. Since we assume the elastomeric matrix to be non-magnetic, the relative permeability of the medium is unity $\left(\mu_{r}=1\right)$. Here $\vec{m}_{i}$ and $\vec{m}_{j}$ are average induced dipole moments of the $i$-th and $j$-th magnetic particles, $\vec{R}_{i j}$ is the radius vector between the $i$-th and $j$-th magnetic particles. The summation in ( 6 ) accounts for the superposition of mutual interaction between all pairs of 
particles in the network. In order to simplify the calculation of the summation, we consider some lattice networks as the representation of the particle's distribution in the matrix. In this work, besides the two lattice models of simple cubic (SC) and body centered cubic (BCC) which have been used in the work of Ivaneyko et al. 2012 [8], face centered cubic (FCC) lattice and edge centered cubic (ECC) have also been investigated. A unit cell of these four lattice networks as models of spatial distribution of the particles is illustrated in Fig. 2.

For the sake of simplicity, all the particles are assumed to have the same spherical shape and with an average radius of $r$. The volume fraction of the magnetic particles dispersed in the matrix is given by:

$$
\phi=\lambda \frac{v_{0}}{a^{3}}
$$

where $v_{0}=\frac{4}{3} \pi r^{3}$ is a particle volume and $\lambda$ is a factor depends on the lattice type which considering Fig. 2 are found to be:

$$
\lambda_{S C}=1, \quad \lambda_{B C C}=2, \lambda_{F C C}=4, \lambda_{E C C}=4
$$

As stated before, application of a magnetic field on a MRE, causes each of the magnetic particles to have a magnetic dipole moment. The induced magnetic dipoles $\vec{m}_{i}$ and $\vec{m}_{j}$ in (6) are directed along the direction of applied magnetic field, $\vec{H}$, toward y-axis as shown in Fig. 1. Because of the symmetry of the lattice models with regard to the magnetic field, the absolute values of dipole moments are identical and related to the magnetization $(M)$ of each particle as $m_{i}=m_{j}=v_{0} M$.

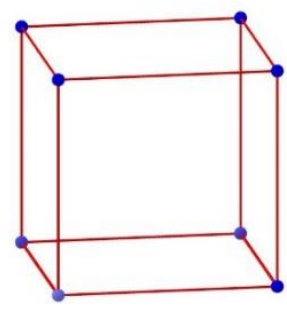

Simple cubic (SC)

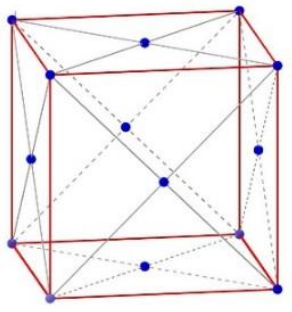

Face centered cubic (FCC)

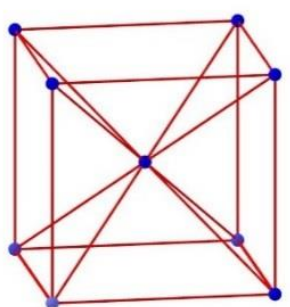

Body centered cubic (BCC)

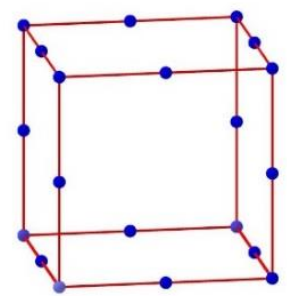

Edge centered cubic (ECC)
Figure 2. Schematic illustration of different lattice networks as representation for spatial distribution of magnetic particles in the elastomeric matrix

The relationship between the particle magnetization $M$ and the applied field strength, $H$, can be approximated by the Frohlish-Kennely equation as [8, 16]:

$$
M=\frac{M_{s}\left(\mu_{i n i}-1\right) H}{M_{s}+\left(\mu_{i n i}-1\right)|H|}
$$

where $M_{s}$ is the saturation magnetization and $\mu_{\text {ini }}$ is the initial magnetic permeability of the particles. To have more compact and simpler formulation, (9) can be transferred into a dimensionless form as:

$$
\frac{M}{M_{S}}=\frac{h}{1+|h|}
$$

where $h=\left(\mu_{\text {ini }}-1\right) H / M_{s}$ is called reduced magnetic field and $M / M_{S}$ the reduced magnetization. Equation (9) can predict saturation of a magnetic material. Indeed, the value of magnetization $M$ increases with an increasing magnetic field and reaches to saturation magnetization, $M_{s}$, when $H \rightarrow \infty$.

By expressing the spatial distribution of particles with simplified arrangements as the lattice models presented in Fig. 2 , the summation over indices $i$ and $j$ in ( 6 ) can be simplified. The $j$-th particle is taken and the summation is performed over index $i$. Since there is a translational symmetry in the infinite lattice with regards to the magnetic field, the dipole-dipole interaction of a given $j$-th particle with adjacent particles around it does not depend on the number $j$. Therefore, the double sum over indices $i$ and $j$ is reduced to a simple sum over index $i$ multiplied by the number of particles $N$. Thus, ( 6 ) can be simplified as:

$$
e_{m}=-u_{0} v_{0}^{2} c\left(\frac{M}{M_{s}}\right)^{2} \sum_{i}\left[\frac{3\left(\vec{R}_{i}\right)_{x}^{2}-\left|\vec{R}_{i}\right|^{2}}{\left|\vec{R}_{i}\right|^{5}}\right]
$$

where $c=N / V$ is the concentration of particles. The vector $\vec{R}_{i j}$ is replaced by $\vec{R}_{i}$ for convenience, since the vector is calculated from the origin of a lattice. The parameter $u_{0}$ is defined as

$$
u_{0}=\frac{\mu_{0} M_{s}^{2}}{4 \pi}
$$

which is defined as the characteristic energy density for magnetic interaction [8]. The radius vector $\overrightarrow{R_{l}}$ in ( 11 ) depends on the macroscopic shear strain $\gamma$. The components of the radius vector after deformation can be defined as

$$
\begin{array}{r}
\left(R_{i}\right)_{x}=\left(R_{i}^{0}\right)_{x} \lambda_{x}=\left(R_{i}^{0}\right)_{x} \frac{\left(\gamma+\sqrt{4+\gamma^{2}}\right)}{2} \\
\left(R_{i}\right)_{y}=\left(R_{i}^{0}\right)_{y} \lambda_{y}=\left(R_{i}^{0}\right)_{y} \\
\left(R_{i}\right)_{z}=\left(R_{i}^{0}\right)_{z} \lambda_{z}=\left(R_{i}^{0}\right)_{z} \frac{2}{\left(\gamma+\sqrt{4+\gamma^{2}}\right)}
\end{array}
$$

where $\left(\vec{R}_{i}^{0}\right)_{\xi}$ are the components of the radius vectors before deformation, $(\xi=x, y, z)$. In a non-deformed MRE, the radius vector $\vec{R}_{i}^{0}$ is defined based on the assumed lattice models in Fig. 2 and can be presented as, 


$$
\vec{R}_{i}^{0}=a \cdot \vec{r}_{l}
$$

where the dimensionless vector $\vec{r}_{l}$ runs over all particles on the sites of all lattices with the edge length $a=1$. Using (10), and (13-16), the magnetic energy $e_{m}$ as a function of a reduced magnetic field $h$ and shear strain $\gamma$ can be expressed as:

$$
e_{m}=u_{0} \phi^{2}\left(\frac{h}{1+|h|}\right)^{2} f(\gamma),
$$

where the dimensionless function of strain $f(\gamma)$ is in the following form:

$$
\begin{aligned}
& f(\gamma) \\
& =-\frac{8}{\lambda}(\Gamma)^{3} \sum_{i}\left[\frac{2 r_{i x}^{2}(\Gamma)^{4}-4 r_{i y}^{2}(\Gamma)^{2}-16 r_{i z}^{2}}{\left(r_{i x}^{2}(\Gamma)^{4}+4 r_{i y}^{2}(\Gamma)^{2}+16 r_{i z}^{2}\right)^{\frac{5}{2}}}\right]
\end{aligned}
$$

and $\Gamma=\gamma+\sqrt{4+\gamma^{2}}$.

The components of vector $\vec{r}_{l}=\left(r_{i x}, r_{i y}, r_{i z}\right)$ represent the location of all particles in the lattices which depend on the lattice type. Accordingly, the value of the function $f(\gamma)$ also depends on the lattice type. In order to solve the problem numerically in the next section, vector $\vec{r}_{l}$ should be programmed as such to locate the particles arranged in the four considered lattice networks. This would be done through nested loops in the coding for each of the lattice types. The number of loops depend on the configuration of particles in the lattice.

Finally, using ( 5 ) for elastic energy and ( 17 ) for magnetic energy, the total potential energy of the deformed MRE under application of the external magnetic field, can be obtained as:

$$
e=\frac{G_{0}}{2}\left(\left(\frac{\Gamma}{2}\right)^{2}+\left(\frac{2}{\Gamma}\right)^{2}-2\right)+u_{0} \phi^{2}\left(\frac{h}{1+|h|}\right)^{2} f(\gamma)
$$

where the value of function $f(\gamma)$ presented in ( 18 ) depends on the lattice types shown in Fig. 2. Having the total energy as a combination of elastic and magnetic contributions, the magneto-mechanical properties of the MRE can be investigated. In the next section, elastic shear modulus of the elastomer will be obtained from the energy function and will be presented on graphs as a function of the applied magnetic field intensity.

\section{B. Elastic Shear Modulus}

The interesting feature of magnetorheological elastomers is their adaptability and possibility of changing their modulus of elasticity by application of an external magnetic field. Shear modulus of a MRE sample having different idealized distribution of particles can be derived from the total potential energy of the material formulated in the previous section. Considering the case of a MRE sample under shear deformation normal to the direction of external magnetic field as shown in Fig. 1, the elastic shear modulus can be obtained as the second derivative of total energy with respect to $\gamma$, at equilibrium state as:

$$
G=\left.\frac{\partial^{2} e}{\partial \gamma^{2}}\right|_{\gamma=0},
$$

where $G$ is the shear modulus of the MRE. Here, the ratio of modulus $G / G_{0}$ is going to be depicted as a function of magnetic field magnitude $H$ for each of the considered types of particle networks shown in Fig. 2. This dimensionless ratio also depends on the parameter $\phi$ and the dimensionless ratio of $G_{0} / u_{0}$.

\section{RESULTS AND DISCUSSIONS}

By programming in MATLAB $^{\circledR}$ environment, shear modulus is calculated numerically. Fig. 3 shows the shear modulus ratio $G / G_{0}$ as a function of the applied magnetic field, $H$, at different volume fractions $\phi=0,0.05,0.1,0.15$ and constant value of $G_{0} / u_{0}=1.5$ for all types of lattices considered in this work i.e. simple cubic, body centered cubic, face centered cubic, and edge centered cubic. Fig. 4 presents the same results at the fixed value of volume fraction $\phi=0.1$ but different values of parameter $G_{0} / u_{0}=1,1.5,5,10$. It is noted that numerical values of $M_{s}=1990 \mathrm{kA} / \mathrm{m}$, and $\mu_{\text {ini }}=132$ as in [16] are utilized for obtaining numerical results.

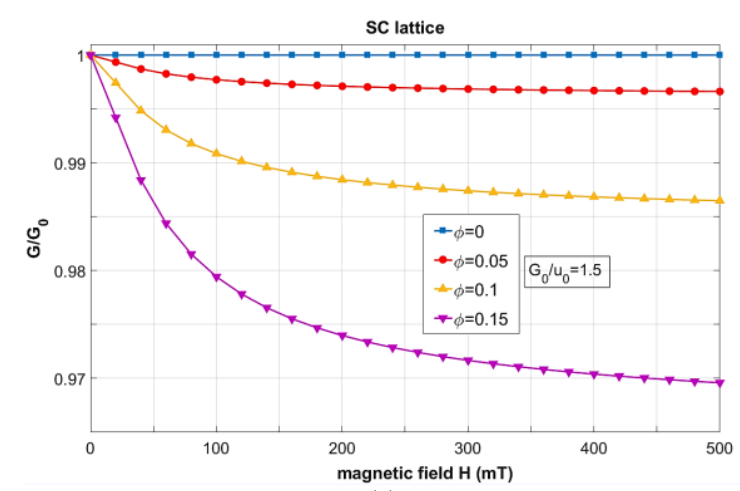

(a)

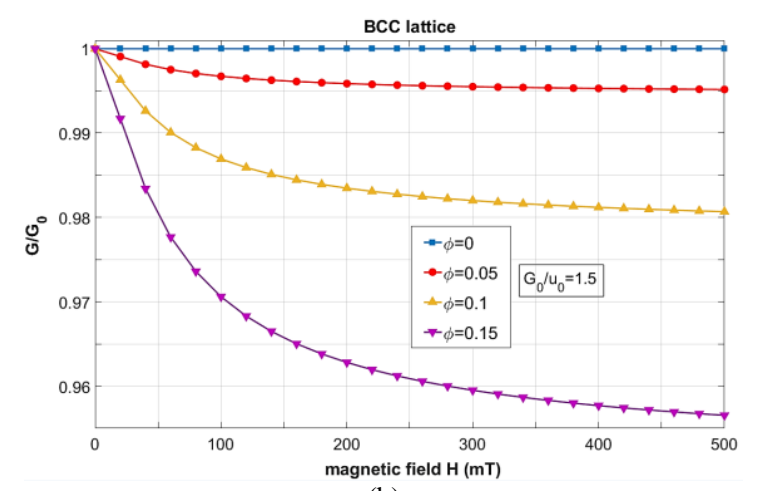

(b) 


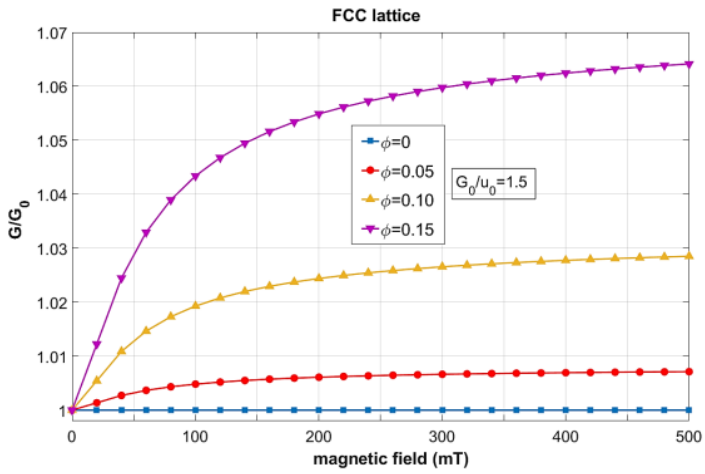

(c)

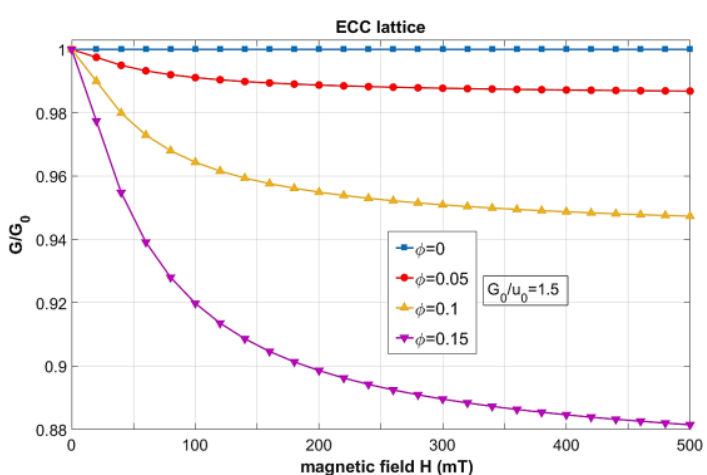

(d)

Figure 3. Shear modulus ratio $\boldsymbol{G} / \boldsymbol{G}_{\mathbf{0}}$ versus magnetic field intensity $\boldsymbol{H}(\mathrm{mT})$ at different volume fractions $\boldsymbol{\phi}$, obtained for four different lattice networks: simple cubic lattice (a), body-centered cubic lattice (b), face centered cubic lattice (c), and edge centered cubic lattice (d).

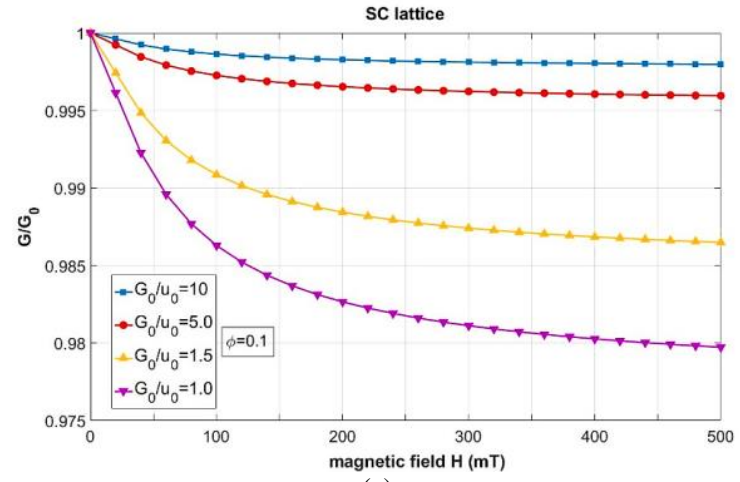

(a)

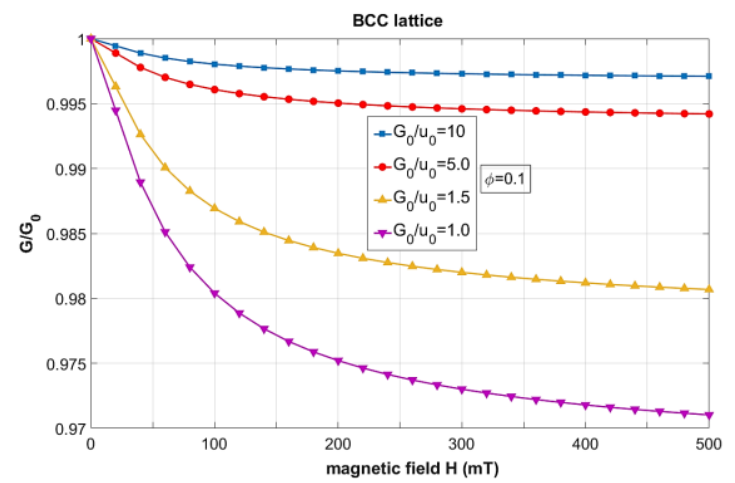

(b)

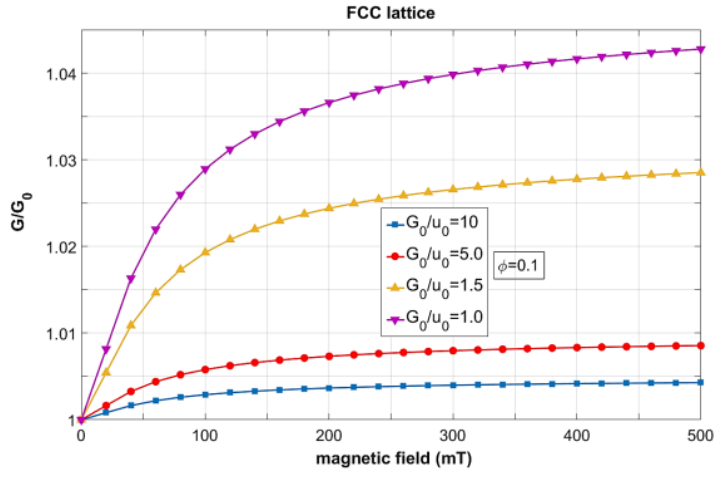

(c)

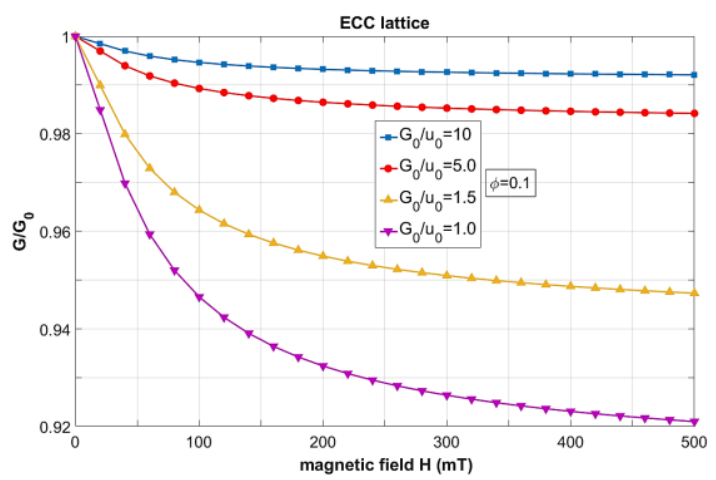

(d)

Figure 4. Shear modulus ratio $G / G_{0}$ versus magnetic field intensity $H(\mathrm{mT})$ at different values of $G_{0} / u_{0}$ and fixed volume fraction $\phi=0.1$, obtained for four types of lattice networks same as Fig. 3

Examination of Figs. 3 and 4 reveals that, shear modulus of a magnetorheological elastomer decreases with magnetic field for the simple cubic, body centered cubic, and edge centered cubic lattices and increases for the face centered cubic lattice. These observations obviously show the impact of particles' network type on the magneto-mechanical behavior of MR elastomers. Different responses of lattice types to the applied magnetic field is due to the special arrangement of particles in each network which causes a different interaction between magnetic particles. Since the mutual interaction of all particles in the network is considered and summed for the calculation of magnetic energy and shear modulus, it is very difficult to evaluate the differences in reciprocal actions of particles for each lattice type. Among the considered lattices, edge centered cubic lattice has the largest change in the absolute value of the change of the shear modulus $\left|G-G_{0}\right|$ with magnetic field. This may be due to the more compact arrangement of particles in this lattice type in comparison to the others.

In addition, it can be seen from Fig. 3 that an increase of the volume fraction $\phi$ results in the increase of the absolute value of the change of the modulus $\left|G-G_{0}\right|$ for all lattice types. That means the more number of particles participated in the material, the bigger the contribution of magnetic energy in the shear modulus by the application of a magnetic field. Furthermore, according to Fig. 4 , increase of the parameter $G_{0} / u_{0}$ decreases the absolute value of the change of the modulus $\left|G-G_{0}\right|$ for all distributions of particles. Considering that the parameter $G_{0} / u_{0}$ 
is in fact the ratio of the characteristic values of the elastic energy to the magnetic energy, increase of this parameter decreases the contribution of magnetic interaction on the shear modulus of the material. The results of this paper are compatible with those reported in [8] for the Young's modulus of a magneto-sensitive elastomer.

\section{CONCLUSIONS}

In this work, a microstructure model of mechanical properties of MR elastomer materials was employed and extended to predict the static shear modulus of a MRE under an external applied magnetic field. To identify the effect of magnetic particles distribution on the magneto-mechanical behavior of a MRE, four idealized homogeneous arrangements of particles called lattice networks were considered. The utilized lattice types are simple cubic, body centered cubic, face centered cubic, and edge centered cubic. First the mathematical formulation of the total potential energy of a MRE sample under a shear deformation and also an external magnetic field was derived. The total energy has two parts of the elastic energy and the magnetic energy. The latter one depends on the interaction of magnetic particles and so on the lattice type. Second the shear modulus of the MR elastomer was extracted from the total energy. Then, using numerical calculations, the shear modulus was obtained as a function of magnetic field and presented for samples having different content of particles and initial properties.

According to the results presented in this work, the shear modulus decreases with increase of the magnetic field for three of the considered lattice types including simple cubic, body centered cubic, and edge centered cubic. However, the shear modulus increases with the magnetic field for the face centered cubic lattice. The variety of the results of the lattice types to the magnetic field is an obvious indication for the impact of magnetic particles distribution on the magneto-mechanical properties of the material. Besides, the effect of volume fraction of particles $\phi$ and the ratio $G_{0} / u_{0}$ on the shear modulus is also investigated. The absolute value of the change of shear modulus from the initial modulus $\left|G-G_{0}\right|$ increases with an increase of volume fraction and decreases with an increase of the ratio $G_{0} / u_{0}$.

In order to know which of the assumed particle networks can be a more realistic model of particles distribution in homogeneous MRE materials, these theoretical results should be compared with experimental tests. Looking through the literature, the static shear modulus of a MR elastomer normally should increases under an applied magnetic field [7, 9, 17]. Accordingly, the results of the face centered cubic (FCC) lattice can be of higher validity. Comparing the theoretical results of the investigated model in the present work with the results of experimental tests can be as an objective of another work in this subject.

\section{REFERENCES}

[1] J. Sutrisno, A. Purwanto, and S.A. Mazlan, "Recent progress on magnetorheological solids: materials, fabrication, testing, and applications," Advanced engineering materials, 2015. 17(5): pp. 563-597.

[2] M.A. Cantera, M. Behrooz, R.F. Gibson, and F. Gordaninejad, "Modeling of magneto-mechanical response of magnetorheological elastomers (MRE) and MRE-based systems: a review," Smart Materials and Structures, 2017. 26(2): pp. 023001.

[3] I. Brigadnov and A. Dorfmann, "Mathematical modeling of magnetosensitive elastomers," International Journal of Solids and Structures, 2003. 40(18): pp. 4659-4674.

[4] I. Agirre-Olabide, A. Lion, and M. Elejabarrieta, "A new threedimensional magneto-viscoelastic model for isotropic magnetorheological elastomers," Smart Materials and Structures, 2017. 26(3): pp. 035021.

[5] M. Norouzi, S.M. Sajjadi Alehashem, H. Vatandoost, Y.Q. Ni, and M.M. Shahmardan, "A new approach for modeling of magnetorheological elastomers." Journal of Intelligent Material Systems and Structures, 2016. 27(8): pp. 1121-1135.

[6] M.R. Jolly, J.D. Carlson, B.C. Muñoz, and T.A. Bullions, "The magnetoviscoelastic response of elastomer composites consisting of ferrous particles embedded in a polymer matrix," Journal of Intelligent Material Systems and Structures, 1996. 7(6): pp. 613-622.

[7] L. Davis, "Model of magnetorheological elastomers," Journal of Applied Physics, 1999. 85(6): pp. 3348-3351.

[8] D. Ivaneyko, V. Toshchevikov, M. Saphiannikova, and G. Heinrich, "Effects of particle distribution on mechanical properties of magnetosensitive elastomers in a homogeneous magnetic field," arXiv preprint arXiv:1210.1401, 2012.

[9] Y. Shen, M.F. Golnaraghi, and G. Heppler, "Experimental research and modeling of magnetorheological elastomers," Journal of Intelligent Material Systems and Structures, 2004. 15(1): pp. 27-35.

[10] J. Ginder and L. Davis, "Shear stresses in magnetorheological fluids: role of magnetic saturation," Applied Physics Letters, 1994. 65(26): pp. 34103412.

[11] J. Ginder, L. Davis, and L. Elie, "Rheology of magnetorheological fluids: models and measurements," International journal of modern physics b, 1996. 10(23n24): pp. 3293-3303.

[12] Y.-s. Zhu, X.-1. Gong, H. Dang, X.-z. Zhang, and P.-q. Zhang, "Numerical analysis on magnetic-induced shear modulus of magnetorheological elastomers based on multi-chain model," Chinese Journal of Chemical Physics, 2006. 19(2): pp. 126.

[13] L.R.G. Treloar, The physics of rubber elasticity, 1975: Oxford University Press. USA.

[14] R.W. Ogden, Non-linear elastic deformations. 1997: Courier Corporation.

[15] R.E. Rosensweig, Ferrohydrodynamics. 2013: Courier Corporation.

[16] G. Bossis, C. Abbo, S. Cutillas, S. Lacis, and C. Metayer, "Electroactive and electrostructured elastomers," International Journal of Modern Physics B, 2001. 15(06n07): pp. 564-573.

[17] C. Bellan and G. Bossis, "Field dependence of viscoelastic properties of MR elastomers," International Journal of Modern Physics B, 2002. 16(17n18): pp. 2447-2453. 